PROCEEDINGS OF THE

AMERICAN MATHEMATICAL SOCIETY

Volume 127, Number 9, Pages 2773-2777

S 0002-9939(99)04836-4

Article electronically published on April 23, 1999

\title{
EXTINGUISHING THE DISTINGUISHED LOGARITHM PROBLEMS
}

\author{
MARK FINKELSTEIN, HOWARD G. TUCKER, AND JERRY ALAN VEEH \\ (Communicated by Stanley Sawyer)
}

\begin{abstract}
The existence, uniqueness, and convergence properties of distinguished logarithms of multivariate characteristic functions are proved.
\end{abstract}

\section{INTRODUCTION}

Weak convergence of probability measures on $\mathbf{R}^{d}$ is often studied by using characteristic functions. Especially in connection with central limit theorems the complex valued characteristic functions never vanish, and convergence of these characteristic functions is established by showing that the logarithms of the characteristic functions converge. In the golden age of this development the twin problems of the existence of, and convergence properties for, these logarithms were often inadequately treated.

More precisely, the two problems are these. First, if $f: \mathbf{R}^{d} \rightarrow \mathbf{C}$ is a characteristic function which never vanishes, does $f$ have a unique continuous logarithm, called the distinguished logarithm, that vanishes at the origin? Second, if $\left\{f_{n}\right\}$ and $f$ are non-vanishing characteristic functions defined on $\mathbf{R}^{d}$ and $\left\{f_{n}\right\}$ converges to $f$, do the distinguished logarithms $\left\{\log \left(f_{n}\right)\right\}$ converge to the distinguished logarithm $\log (f)$ ?

These problems for probability measures on the real line were addressed some 30 years later. Chung [2, pages 221-222] laments on this situation for the existence problem as follows:

"Although merely an exercise in 'complex variables', it has been treated in the existing literature in a cavalier fashion and then misused or abused. For this reason the main propositions will be spelled out in meticulous detail here."

Chung then provides a complete proof of the existence and uniqueness of logarithms of non-vanishing characteristic functions by a method he calls "continuous continuation" and also proves a convergence theorem. His proof relies heavily on the order properties of the real line. A similar approach was used by Chow and Teicher [1] to address these problems. Tucker [4] obtains similar results by a different method. In all cases the order properties of the real line play a key role in the proofs.

Received by the editors July 14, 1997 and, in revised form, December 1, 1997.

1991 Mathematics Subject Classification. Primary 60E10, 60E07, 60F05.

Key words and phrases. Distinguished logarithms, characteristic functions, infinitely divisible laws, logarithms. 
These problems for characteristic functions defined over $\mathbf{R}^{d}, d>1$, require a different method which does not rely on the order properties of the underlying space. This paper solves the two problems in this general setting by developing a new method. This method provides an alternate approach to the problems in the case $d=1$.

\section{THE THEOREMS}

The two theorems proved here involve the existence of, and convergence properties for, the logarithms of characteristic functions of probability measures on $\mathbf{R}^{d}$. The proofs reveal that these theorems are actually valid in a slightly wider context.

Theorem 1. If $f: \mathbf{R}^{d} \rightarrow \mathbf{C}$ is a continuous function which is never 0 and which satisfies $f(0)=1$, then there is a unique continuous function $\varphi: \mathbf{R}^{d} \rightarrow \mathbf{R}^{1}$ which satisfies

(1) $\varphi(0)=0$

(2) $\varphi$ is continuous, and

(3) $f(x)=|f(x)| e^{i \varphi(x)}$ for all $x \in \mathbf{R}^{d}$.

The unique function $\log (|f(x)|)+i \varphi(x)$ determined from $f$ by Theorem 1 will be referred to as the distinguished logarithm of $f$ and denoted by $\log (f)$.

Theorem 2. If $f$ and $\left\{f_{n}\right\}$ are continuous functions mapping $\mathbf{R}^{d}$ to $\mathbf{C}$ which are never 0 and which satisfy $f(0)=f_{n}(0)=1$ for all $n$ and if $\left\{f_{n}\right\}$ converges uniformly to $f$ on compact subsets of $\mathbf{R}^{d}$, then the sequence of distinguished logarithms $\left\{\log \left(f_{n}\right)\right\}$ also converges uniformly on compact subsets of $\mathbf{R}^{d}$ to the distinguished logarithm $\log (f)$.

These two theorems also have local versions which may be deduced from the proofs. These local versions replace $\mathbf{R}^{d}$ in the statement of the theorems by a closed ball $D$ centered at 0 with an arbitrary positive radius.

\section{THE PROOFS}

Lemma 1. The conclusion of Theorem 1 holds under the additional assumption that $f$ has continuous first partial derivatives.

Proof. The idea of the proof is to define the logarithm of $f$ in a way that is path independent. The allowable paths are the images under $f$ of paths in $\mathbf{R}^{d}$, not arbitrary paths in $\mathbf{C}$. For the first part of the proof let $x \in \mathbf{R}^{d}$ be fixed. Let $r:[0,1] \rightarrow \mathbf{R}^{d}$ be a piecewise continuously differentiable mapping which satisfies $r(0)=0$ and $r(1)=x$. Let $\gamma(t)=f(r(t))$ be the corresponding path connecting 1 and $f(x)$ in $\mathbf{C}$. Because of the assumptions on the smoothness of $f$ and $r, \gamma$ is a rectifiable curve. Define $G: \mathbf{R}^{d} \rightarrow \mathbf{C}$ by

$$
G(x)=\int_{\gamma} \frac{1}{z} d z
$$

so that $e^{G(x)}=f(x)$. Then set $\varphi(x)=\frac{1}{i}(G(x)-\log |f(x)|)$. Note that $G(x)$ is a candidate for the distinguished logarithm $\log (f)$.

The first claim is that $\varphi$ is independent of the path $r$ in $\mathbf{R}^{d}$ which connects 0 and $x$. This will be proved by showing that $G$ is independent of the path $r$ in $\mathbf{R}^{d}$ which connects 0 and $x$. Let $\tilde{r}$ be another piecewise smooth path in $\mathbf{R}^{d}$ which connects 
0 and $x$ and let $\tilde{\gamma}(t)=f(\tilde{r}(t))$. The independence of $\varphi$ and the path will follow provided that

$$
\int_{\gamma} \frac{1}{z} d z=\int_{\tilde{\gamma}} \frac{1}{z} d z
$$

Now $1 / z$ is analytic in $\mathbf{C} \backslash\{0\}$ so the equality of these two integrals follows from Cauchy's Theorem if the two paths $\gamma$ and $\tilde{\gamma}$ are homotopic in $\mathbf{C} \backslash\{0\}$. The mapping $h:[0,1] \times[0,1] \rightarrow \mathbf{C}$ defined by $h(s, t)=f(s r(t)+(1-s) \tilde{r}(t))$ provides the necessary homotopy, since $f$ never vanishes and both $r$ and $\tilde{r}$ are continuous. This proves the first claim.

The second claim is that $\varphi$ is a continuous function. This property of $\varphi$ will be established by showing that $G$ is continuous. For $x$ and $y$ in $\mathbf{R}^{d}$ let $r_{x}(t)$ and $r_{y}(t)$ be the straight line paths connecting 0 to $x$ and $y$ respectively. Let $r_{x, y}(t)$ be the straight line path connecting $x$ to $y$ in $\mathbf{R}^{d}$. Denote by $\gamma_{x}, \gamma_{y}$, and $\gamma_{x, y}$ the respective images of these paths under $f$. Since $G$ does not depend on the path in $\mathbf{R}^{d}$

$$
|G(x)-G(y)|=\left|\int_{\gamma_{x}} \frac{1}{z} d z-\int_{\gamma_{y}} \frac{1}{z} d z\right|=\left|\int_{\gamma_{x, y}} \frac{1}{z} d z\right| .
$$

Now let $D$ be a closed ball in $\mathbf{R}^{d}$ centered at 0 which has $x$ and $y$ in its interior. Since $f$ is continuous and does not vanish, $0<\eta \equiv \min \{|f(w)|: w \in D\}$. Also since the partial derivatives $D_{j} f$ of $f$ are assumed to be continuous, $M \equiv \max \left\{\left|D_{j} f(w)\right|\right.$ : $1 \leq j \leq d, w \in D\}<\infty$. Direct estimation of the last integral above gives

$$
\begin{aligned}
|G(x)-G(y)| & =\left|\int_{\gamma_{x, y}} \frac{1}{z} d z\right| \\
& \leq \int_{\gamma_{x, y}}\left|\frac{1}{z}\right||d z| \leq \frac{1}{\eta} \int_{\gamma_{x, y}}|d z| \leq \frac{M d}{\eta}\|x-y\| .
\end{aligned}
$$

This shows that $G$ is continuous. This concludes the proof of the second claim. Since $G(0)=0$ the proof is concluded except for the uniqueness assertion. If $\varphi$ and $\tilde{\varphi}$ are two functions satisfying the conclusions of the theorem, then $\varphi-\tilde{\varphi}$ is a continuous function which vanishes at 0 and takes values which are integer multiples of $2 \pi$. Thus $\varphi=\tilde{\varphi}$ and the proof of the lemma is concluded.

The proof of Lemma 1 has actually established a somewhat different result which will be used shortly. If the function $f$ is smooth and does not vanish on a closed ball $D \subset \mathbf{R}^{d}$ centered at 0 , then $f$ has a distinguished logarithm defined on the interior of $D$. This readily follows from the fact that the above arguments used only the local behavior of $f$.

Lemma 2. If $g: \mathbf{R}^{d} \rightarrow \mathbf{C}$ is continuous, there is a sequence $\left\{g_{n}\right\}$ of $C^{\infty}$ functions $g_{n}: \mathbf{R}^{d} \rightarrow \mathbf{C}$ which converges uniformly to $g$ on compact subsets of $\mathbf{R}^{d}$ and which satisfy $g_{n}(0)=g(0)$ for all $n$.

Proof. The existence of the smooth uniformly convergent sequence is proved on pages 55-56 of Donoghue [3]. The fact that this sequence can be chosen so that $g_{n}(0)=g(0)$ for all $n$ then follows easily.

Proof of Theorem 1. Let the positive radius of a closed ball $D \subset \mathbf{R}^{d}$ centered at 0 be fixed but arbitrary and let $\eta_{D}=\min \{|f(x)|: x \in D\}$. Note that $\eta_{D}>0$ since 
$f$ is continuous and never vanishes. Use Lemma 2 to obtain a sequence $\left\{f_{n}\right\}$ of smooth functions which converge uniformly to $f$ on $D$. There is an integer $N_{D}>0$ with the property that $\left|f_{n}(x)-f(x)\right|<\eta_{D} / 8$ and $\left|f_{n}(x)-f_{m}(x)\right|<\eta_{D} / 4$ for all $x \in D$ and for all $n$ and $m$ exceeding $N_{D}$. Denote by $G_{n}$ and $\varphi_{n}$ the functions constructed as in the proof of Lemma 1 starting with $f_{n}$ and $D$ and using the remark following the proof of Lemma 1. Note that this construction may only be valid for $n>N_{D}$ since it is only for such $n$ that $f_{n}$ is known not to vanish on $D$.

The objective is to show that $\left\{G_{n}\right\}$ is uniformly Cauchy on $D$. This will imply that $G(x)=\lim _{n \rightarrow \infty} G_{n}(x)$ exists and is continuous on $D$. From this $G$ the corresponding $\varphi$ can be obtained as before and the desired distinguished logarithm of $f$ over $D$ obtained. Since the radius of $D$ is arbitrary the existence of a distinguished logarithm of $f$ over $\mathbf{R}^{d}$ follows.

To obtain this uniform Cauchy property let $x \in D$ be fixed and let $r(t)$ be the straight line path connecting 0 and $x$ in $\mathbf{R}^{d}$. Define $\gamma_{n}(t)=f_{n}(r(t))$. Also let $\gamma_{n, m}(t)=(1-t) f_{n}(x)+t f_{m}(x)$ be the straight line path in $\mathbf{C}$ connecting $f_{n}(x)$ and $f_{m}(x)$. To estimate $\left|G_{n}(x)-G_{m}(x)\right|$ Cauchy's Theorem will be applied as before.

Justification of the use of Cauchy's Theorem demands that the path of integration be homotopic to a point, the homotopy being with respect to the punctured complex plane. For each $n, m>N_{D}$ and each $y \in D$ and for $0 \leq t \leq 3$ define

$$
\gamma(t, y)= \begin{cases}f_{n}(t y) & \text { if } 0 \leq t \leq 1 \\ (2-t) f_{n}(y)+(t-1) f_{m}(y) & \text { if } 1 \leq t \leq 2 \\ f_{m}((3-t) y) & \text { if } 2 \leq t \leq 3\end{cases}
$$

To see that this path is homotopic to a point over the punctured complex plane define $h:[0,1] \times[0,3] \rightarrow \mathbf{C}$ by $h(s, t)=\gamma(t, s y)$. Then $h$ is clearly a homotopy which collapses the path $\gamma(t, y)$ to a point. Simple estimates using the triangle inequality and the fact that $|f(x)|>\eta_{D},\left|f_{n}(x)-f(x)\right|<\eta_{D} / 8$ and $\left|f_{n}(x)-f_{m}(x)\right|<\eta_{D} / 4$ for all $x \in D$ and for all $n$ and $m$ exceeding $N_{D}$ show that $h(s, t) \in\{z \in \mathbf{C}:|z|>$ $\left.\eta_{D} / 2\right\} \subset \mathbf{C} \backslash\{0\}$. This proves the claim.

To continue the proof of the uniform Cauchy behavior of the sequence $\left\{G_{n}(x)\right\}$ over $D$ now estimate

$$
\begin{aligned}
\left|G_{n}(x)-G_{m}(x)\right| & =\left|\int_{\gamma_{n}} \frac{1}{z} d z-\int_{\gamma_{m}} \frac{1}{z} d z\right|=\left|\int_{\gamma_{n, m}} \frac{1}{z} d z\right| \\
& \leq \int_{\gamma_{n, m}}\left|\frac{1}{z}\right||d z| \leq \frac{8}{3 \eta_{D}} \int_{\gamma_{n, m}}|d z| \\
& \leq \frac{8}{3 \eta_{D}}\left|f_{n}(x)-f_{m}(x)\right|
\end{aligned}
$$

since on $\gamma_{n, m}$ the inequality $|z|>3 \eta_{D} / 8$ holds. Thus, the uniform Cauchy behavior of $\left\{G_{n}\right\}$ follows from the uniform convergence of $\left\{f_{n}\right\}$.

Proof of Theorem 2. Apply Lemma 2 to obtain, for each $n$, a sequence $\left\{f_{n, j}: j \geq\right.$ 1 \} of smooth functions which converges uniformly to $f_{n}$ over compact subsets of $\mathbf{R}^{d}$. Let $D$ be a closed disk centered at $0 \in \mathbf{R}^{d}$ with fixed positive radius. Define $\eta_{D}=\min \{|f(x)|: x \in D\}$. For $n$ sufficiently large $\left|f_{n}(x)-f(x)\right|<\eta_{D} / 8$ for all $x \in D$. For each such $n$ there is an integer $j_{n}$ so that $\left|f_{n, j}(x)-f_{n}(x)\right|<$ $\eta_{D} / 2^{n+3}$ for all $x \in D$ and all $j \geq j_{n}$. Now the functions $\left\{f_{n, j_{n}}\right\}$ are smooth, non-vanishing, and converge to $f$ uniformly over $D$. As in the proof of Theorem 
1 the distinguished logarithms $\left\{G_{n, j_{n}}\right\}$ are uniformly Cauchy over $D$ and converge uniformly to the distinguished logarithm $G$ of $f$. The last inequality in the proof of Theorem 1 shows that for the distinguished logarithm $G_{n}$ of $f_{n}$ the inequality $\left|G_{n, j_{n}}(x)-G_{n}(x)\right| \leq 2^{-n} / 3$ holds uniformly over $D$ for all large $n$. Hence, $G_{n} \rightarrow G$ uniformly on $D$.

\section{REFERENCES}

[1] Y. S. Chow and H. Teicher, Probability Theory: Independence, Interchangeability, Martingales, Second Edition, Springer Verlag, New York, 1988. MR 89e:60001

[2] K. L. Chung, A Course in Probability, First Edition, Harcourt, Brace and World, New York, 1968. MR 37:4842

[3] W. F. Donoghue, Jr., Distributions and Fourier Transforms, Academic Press, New York, 1969.

[4] H. G. Tucker, A Graduate Course in Probability, Academic Press, New York, 1968. MR 36: 4593

(M. Finkelstein and H. G. Tucker) Department of Mathematics, University of California Irvine, Irvine, California 92697-3875

E-mail address: mfinkels@uci.edu

E-mail address: htucker@math.uci.edu

(J. A. Veeh) Department of Mathematics, Auburn University, Auburn, Alabama 368495307

E-mail address: veehjer@mail.auburn.edu 